\title{
Basic essentials: why elderly skin requires special treatment
}

\begin{abstract}
he promotion and maintenance of skin integrity is one of the most common problems faced by staff in any care setting, and the frequent combination of elderly skin and incontinence can be a particularly challenging one. Not surprisingly a large proportion of time and effort is spent on interventions aimed at preventing skin breakdown and restoring skin barrier function. Unfortunately, in many cases the care provided is based more on ritual and tradition rather than any firm evidence or protocol. This situation is further complicated by the ever growing number of skin care products on the market, each with their own claims of efficacy, making it difficult for appropriate choices to be made. However by considering the age-related changes in the skin, and the specific threats posed by incontinence, interventions can be provided that promote effective care and improve the patient experience. In many cases these can be built into existing routines used to meet an individual's personal care needs.
\end{abstract}

\section{Beneath the layers}

One of the major functions of healthy skin is the maintenance of a physical barrier against the external environment. This prevents the entry of harmful substances and pathogens, as well as preventing excessive fluid loss from the body. This is achieved by the uppermost layer of the skin, the epidermis and in particular the outermost part of the epidermis, the stratum corneum (Figure 1). Elderly skin has a thinner overall epidermis; with a flattened interface between the epidermis (upper layer) and dermis (lower

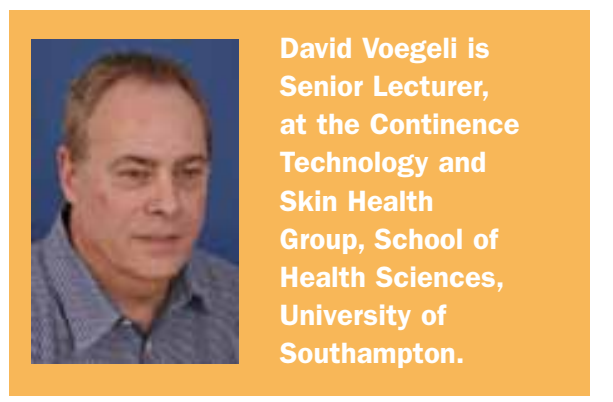

\section{Don't let bad habits and daily rituals take their toll on the skin of your residents. \\ How soap and roughly \\ laundered towels can be

abrasive, and when to use
emollients and creams.

layer) making it less resistant to shearing forces. Also the stratum corneum layers are reduced, decreasing the effectiveness of the skin barrier. With age the complex biochemistry of the dermis is altered, leading to a decrease in collagen synthesis and a progressive loss of elastin, again making the skin less resistant to shearing forces and friction (Voegeli, 2003). The delicate balance between the enzymes that control remodel- ling and repair of the dermal matrix is also disrupted with increasing age, contributing to the overall loss of connective tissue and atrophy of the skin (Millis et al, 1992). Coupled with a decreased healing potential and reduced immune function, it is not surprising that the elderly are at increased risk and that even the simplest insult may precipitate skin breakdown (Waller and Maibach, 2005).

\section{Weak practice}

Unfortunately the skin care provided in health and social care settings tends to be dictated more by habit and ritual, rather than based on the best evidence available (Jeter and Lutz 1996). This presents particular challenges and increases the demand for clearer guidance on best practice to promote skin health. Where evidence does exist it still tends to be fairly weak making definitive recommendations for practice difficult to make (Hodgkinson et al, 2007). Despite this situation, a model of the essen-

\section{Figure 1. Diagramatic representation of the skin structure}

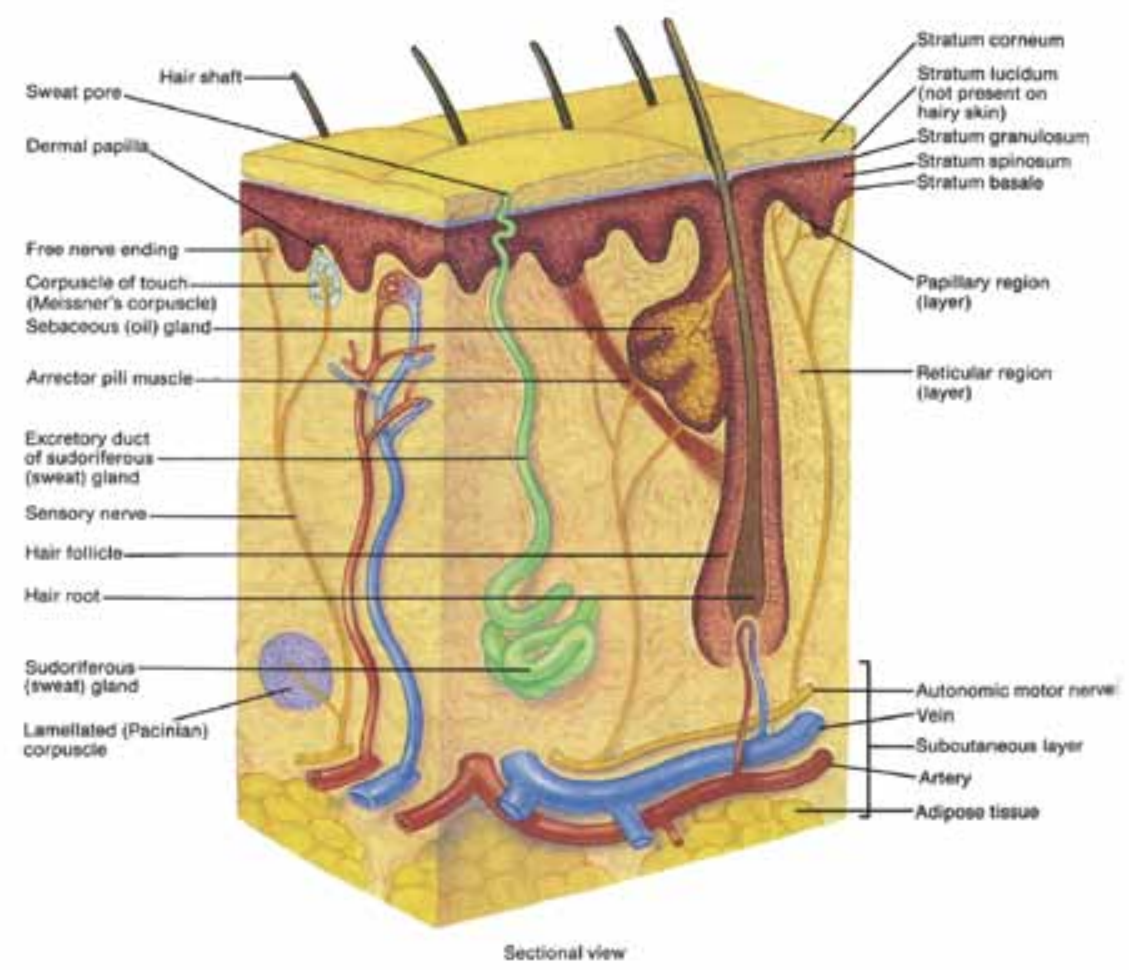


Figure 2. 'Bricks and mortar' model of the stratum corneum

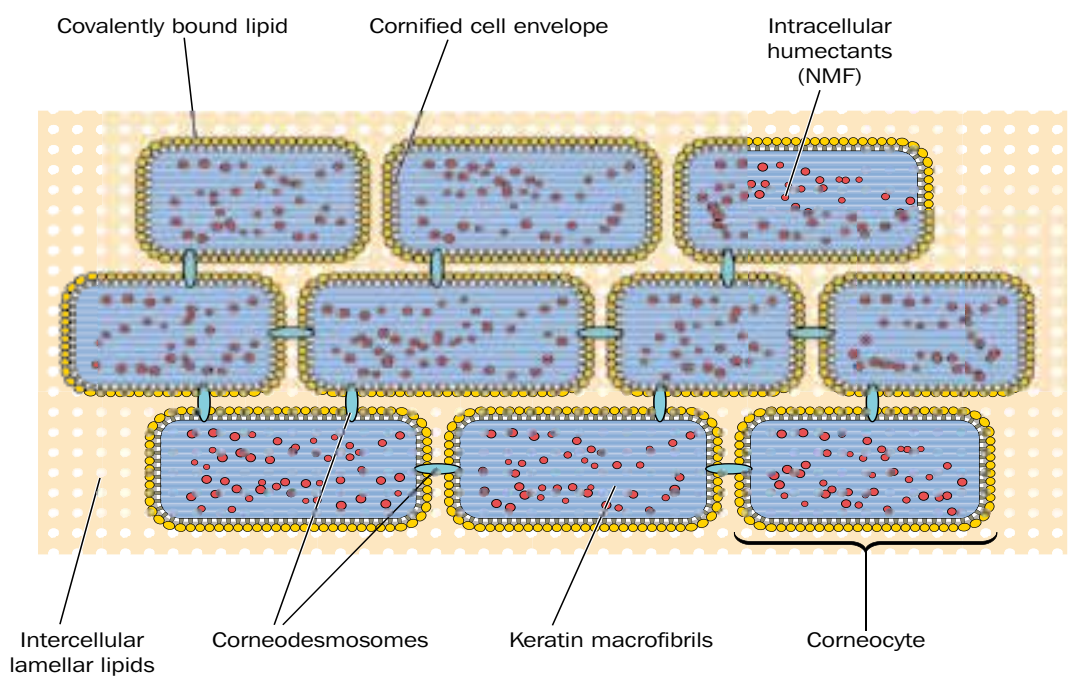

tial components that should be considered when devising a skin care protocol can be devised (Johnson,2004: Figure 2). This suggests that basic skin care consists of four main areas, namely: cleansing; hydrating / moisturizing; protection, and replenishing.

\section{Skin cleansing:}

The main approaches to protecting the skin and helping to prevent skin breakdown remain the appropriate washing and drying of the skin, and the use of barrier therapies. Standard skin care in most care settings consists of washing with soap and water using a flannel or disposable wash cloths, and towel drying either by rubbing or patting. Although this approach may be suitable for protecting the skin from the effects of occasional incontinence or soiling, it does expose the skin to several potentially damaging factors, which may do more harm than good with frequent use.

Soaps are water-soluble sodium or potassium salts of fatty acids that have been treated with a strong alkali, and act as surfactants (Abbas et al 2004). Additional synthetic surfactants, such as sodium lauryl sulphate (SLS), may be added to soap to make it a better wetting agent. However, these synthetic surfactants are known to be potent skin irritants. Further irritation can be caused by the combination of soap and 'hard' water, which produces a precipitate that remains on the surface of the skin if not rinsed off effectively (Timby 1996). Soaps may also adversely affect the skin by caus- ing the excessive removal of natural oils, precipitating drying of the skin, with these effects being aggravated if the water is too hot (Baillie and Arrowsmith 2001).

Due to their highly alkaline nature, the repeated use of soap may shift the $\mathrm{pH}$ of the skin surface making it more alkaline, thereby negating the protective influence of the acid mantle, and upsetting the balance of resident flora on the skin (Korting and Braun-Falco 1996). This may enhance the risk of skin colonization by potentially pathogenic micro-organisms, which may ultimately invade the skin should the barrier function be further disturbed.

It is important that the skin is carefully dried after washing, to avoid maceration, undue cooling, and to maintain patient comfort. The process of towel drying may increase any skin damage occurring during washing by causing direct mechanical injury to the stratum corneum, particularly if rough, commercially laundered towels are used. Ultimately, this may lead to a disruption of the barrier function of the skin, triggering inflammation and increasing the likelihood that the skin will breakdown.

Traditionally, vulnerable skin has been dried by patting rather than rubbing. However, it is difficult to determine whether any one method is preferable to another due to the limited information on the subject. Although a recent study suggests that pat drying with a towel leaves the skin significantly wetter, therefore increasing the risk of overhydration of the stratum corneum, and maceration (Voegeli, 2008). The increased wetness may also expose the skin to a higher risk of friction damage, due to the wet skin sticking to clothing or bedclothes, although this has yet to be conclusively demonstrated.

A number of skin cleansers have been developed to provide an alternative to

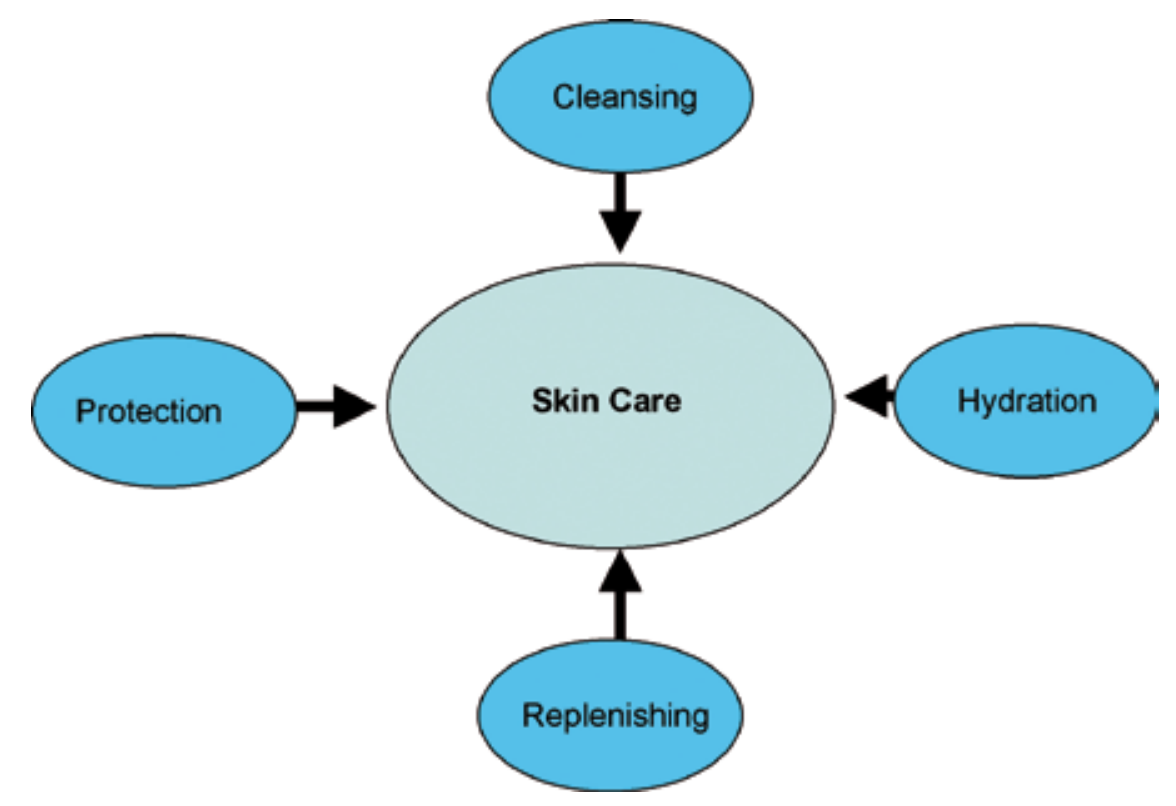

Figure 3. Model of the basic components of skin care. Adapted from Johnson (2004) 
the use of soap and water, with many not needing to be rinsed off after use. They are formulated to be gentler on the skin and help to maintain a $\mathrm{pH}$ level that minimises barrier disruption.

The range of cleansers has increased and become more sophisticated; with the emergence of a small range of products that are designed to cleanse, moisturize and apply a barrier product all in one go (e.g. Tena Wash Cream, Triple Care, ComfortShield). Although they may provide a less irritant means to cleanse the skin, compared to soap and water they are relatively expensive and so are often not used.

\section{Barrier products.}

Barrier creams, ointments and more recently films have traditionally been used to protect the skin from damage caused by excessive exposure to water and irritants (e.g. urinary and faecal incontinence).

However they are sometimes confused with emollients, and although some barrier creams and ointments will have a mildly hydrating effect on the skin, this is not their major action and should not be substituted for an emollient where one is indicated. Similarly, an emollient should not be substituted for a barrier product, as the two have completely different actions. Basic barrier preparations consist of a lipid/ water emulsion base with the addition of metal oxides (e.g. zinc or titanium) which form a thin layer on the surface of the skin to repel potential irritants (e.g. Sudocrem, Table 1). The more sophisticated ones often contain a water repellent silicone-based ingredient such as demeticone, as well as mild antiseptic agents such as cetrimide or benzalkonium.

Unfortunately there is the potential for some of these ingredients to cause irritation in sensitive individuals, with even seemingly innocuous preparations such as zinc and castor oil cream containing arachis (peanut) oil. This should always be kept in mind, particularly if skin irritation appears to worsen when using any preparation.

Advances in polymer science have led to the development of a new generation of product, which allow a thin semi-permeable protective silicone-based polymer coating to be applied to the skin (Cavilon, Skin-Prep, LBF). It would appear that in some situations these polymers have an advantage

Table 1.

NET COST (PER 50G) BARRIER PRODUCTS LISTED IN THE BRITISH NATIONAL FORIMULARY 59

Zinc Cream, BP

Zinc Ointment, BP

Zinc and Castor

Oil Ointment, BP

Conotrane $^{\circledR}$

(cream)

Drapolene $^{\circledast}$

(cream)

Medicaid $^{\circledast}$

(cream)

Metanium $^{\circledast}$

(ointment)

Morhulin $^{\circledR}$

(ointment)

Siopel ${ }^{\circledR}$

(cream)

Sprilon ${ }^{\circledR}$

(spray)

Sudocrem ${ }^{\circledast}$

(cream)

Vasogen ${ }^{\circledR}$

(cream)
Zinc oxide, arachis oil, calcium hydroxide, oleic acid, wool fat, water

Zinc oxide, wool fat, hard paraffin, cetostearyl alcohol, white soft paraffin

Zinc oxide, castor oil, arachis oil, white beeswax, cetostearyl alcohol

Benzalkonium chloride, dimeticone, cetostearyl alcohol, fragrance

Benzalkonium chloride, cetrimide, white soft paraffin, cetyl alcohol, wool fat, chlorocresol

Cetrimide, light liquid paraffin, white soft paraffin, cetostearyl alcohol, glyceryl

Titanium dioxide, titanium peroxide, titanium salicylate, demeticone, light liquid paraffin, white soft paraffin, benzoin tincture

Cod-liver oil, zinc oxide, liquid paraffin, yellow soft paraffin, wool fat derivatives Dimeticone, cetrimide, arachis oil, 2.15 cetostearyl alcohol, hydroxybenzoates, butylated hydroxytoluene

Dimeticone, zinc oxide, wool alcohols, dextran, white soft paraffin, liquid paraffin, hydroxybenzoates, cetostearyl alcohol, wool fat

Benzyl alcohol, benzyl benzoate, benzyl cinnamate, hydrous wool fat, zinc oxide

Dimeticone, calamine, zinc oxide, hydroxybenzoates, wool fat
0.50

From: Joint Formulary Committee (2010) over more conventional products, by saving staff time and offering greater protection against repeated incontinence. Certainly these products have grown in popularity, although they remain fairly expensive.

Concern has been expressed that the use of barrier products, particularly greasy creams and ointments might 'clog' incontinence pads, leading to pad failure and leak- age, a problem that can certainly occur with the overuse of talcum powder.

Once again this is an area that has not been adequately studied, however Bolton et al (2004) suggest that if applied sparingly, according to manufacturers' instructions, most barrier products are safe to use in combination with incontinence pads and do not significantly affect pad performance. 


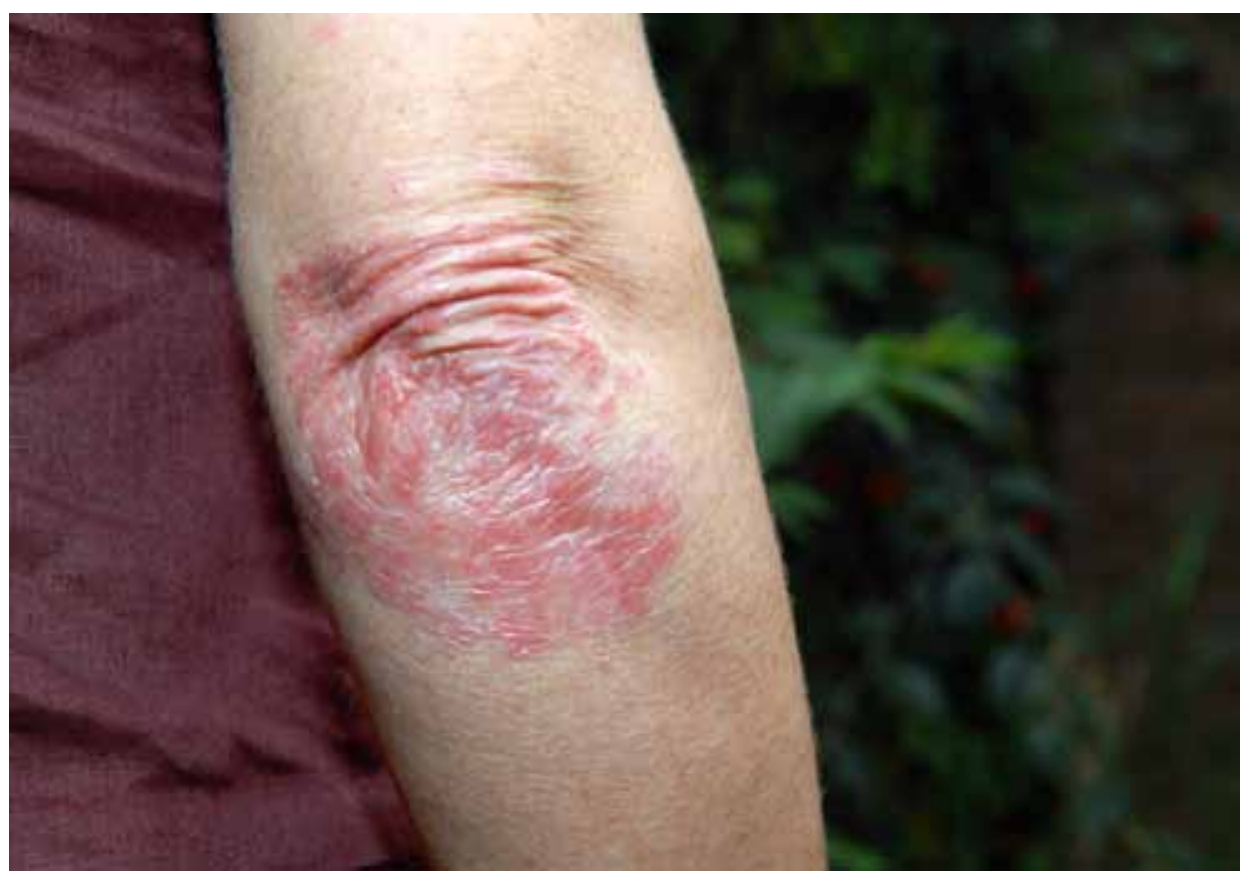

\section{Emollients.}

The term emollient is derived from the Latin meaning to soften, and implies a substance that acts to smooth the skin surface. Commonly the terms emollient and moisturizer are often interchanged as they perform similar functions in terms of increasing hydration of the top layer of the epidermis, the stratum corneum.

Traditionally basic emollients, such as petrolatum, have worked by creating an inert barrier over the skin surface, trapping moisture underneath (Holden et al, 2002). Current emollients are available in the form of sprays, lotions, creams and ointments (Table 2). Although the development and formulation of emollients has moved forward, the basic principle remains the same, namely they are all variations of an oil (lipid) and water emulsion.

The rich mixture of lipid and water makes an ideal breeding ground for bacteria, so in many cases agents to inhibit bacterial growth are needed (e.g. Benzalkonium chloride, hydroxybenzoates).

Occasionally, sensitivity to emulsifying agents, preservatives and other additives (Table 3) may occur worsening the skin irritation and leading to a contact dermatitis (Fan et al, 1999). Although greasier products (ointments) are thought to be more clinically effective, many patients dislike these and find the feel of them, combined with the staining of clothing and bedding they cause, unacceptable. Preference is generally expressed for rapidly absorbed lotions and creams, particularly if being used on visible parts of the body (Holden et al, 2002).

\section{Putting knowledge into practice.}

As the range of products available is increasing, with each one claiming to be more effective than another, it is not surprising that skin care interventions may vary from area to area, and may not necessarily be based on the best available evidence.

One way forward is to adopt the guidelines that have emerged from a growing evidence base of expert clinical opinion (Table 4). These can be utilized to reflect on current care and assist in the development of clinical guidelines and the formulation of skin care protocols.

The first step in any protocol should be the assessment of the patient's skin each time care is carried out, and the accurate documentation of skin integrity. This should be coupled with an assessment of any incontinence, and advice sought from a specialist nurse if necessary.

The potential benefits to patients of adopting clear skin care protocols have been highlighted by several studies based both in the UK and North America. Bale et al (2004) reported that the introduction of a protocol for elderly patients with

\section{Table 2.}

\section{EXAMPLES OF EMOLLIENTS LISTED IN THE BRITISH NATIONAL FORIMULARY 59}

Aveeno

Balneum

Diprobase

Doublebase

E45

Elidel

Epaderm

Hydromol

Lipobase

Oilatum
Endogenous and exogenous eczema, xeroderma, ichthyosis and senile pruritus associated with dry skin

For dry skin conditions (e.g. dermatitis and eczema)

For dry skin conditions

Dry, chapped, or itchy skin conditions

Cream: dry skin conditions

Emollient wash cream: eczema, xeroderma, ichthyosis and senile pruritus associated with dry skin

Lotion: for symptomatic relief of dry skin conditions

Short-term treatment of mild to moderate atopic eczema

For use as an emollient or soap substitute

Cream: for dry skin conditions

Ointment: for use as an emollient, bath additive,

or soap substitute

For dry skin conditions, also for use during treatment with topical corticosteroid and as diluent for Locoid Lipocream

Cream: for dry skin conditions

Shower emollient: for dry skin conditions including dermatitis
From: Joint Formulary Committee (2010) 
Table 3:

\section{POTENTIAL SENSITIZERS IN EMOLLIENTS AND BARRIER PRODUCTS.}

Beeswax

Benzyl alcohol

Butylated hydroxyanisole

Butylated hydroxytoluene

Cetostearyl alcohol (including cetyl and

stearyl alcohol)

Chlorocresol

Edetic acid (EDTA)

Ethylenediamine

Fragrances

Hydroxybenzoates (parabens)

Imidurea

Isopropyl palmitate

$\mathrm{N}$-(3-Chloroallyl)hexaminium chloride

(quaternium 15)

Polysorbates

Propylene glycol

Sodium metabisulphite

Sorbic acid

Wool fat and related substances including Ianolin

Joint Formulary Committee (2010)

incontinence in a nursing home led to an overall improvement in skin health and integrity. Whilst Cole and Nesbitt (2004) and Lyder et al (2002) demonstrated a significant reduction in pressure ulcer incidence rates in both hospital and residential care settings following the implementation of skin care protocols.

In Cole and Nesbitt's case the pressure ulcer incidence rate fell from $17.9 \%$ to $2 \%$ over a three year period, and Lyder's group reporting a remarkable $87 \%$ reduction in incidence rate in one nursing home. These studies demonstrate that small changes, supported by ongoing education, can achieve considerable results in terms of improving skin health in the elderly.

\section{Conclusion}

The high incidence of incontinence in the elderly, particularly those in long-term settings means they are at an increased risk of developing incontinence associated dermatitis and skin breakdown. However, skin care remains a fundamental, yet largely neglected aspect of quality care. The apparent invisibility of this aspect of care means it is often overlooked, yet the importance of effective skin care interventions in reducing the suffering caused by skin breakdown cannot be underestimated. Investment in this area of practice may improve the patient experience, and increasingly evidence is emerging demonstrating that good skin care not only improves clinical outcomes and quality of life, but can also reduce costs, a highly fashionable topic for today's care providers.

NRC

Abbas S, Weiss Goldberg J, Massaro M. (2004) Personal cleanser technology and clinical performance. Dermatologic Therapy.; 17: 35-42.

Baillie, L., Arrowsmith, V. (2001) Meeting elimination needs. Developing Practical Nursing Skills, Ch. 6. Hodder Arnold, London.

Bale S. Tebble N. Jones V. Price P. (2004) The benefits of implementing a new skin care protocol in nursing homes. Journal of Tissue Viability. 14(2), 44-50.

BNF 59 (2010) BMJ Publishing Group Ltd and RPS

Publishing, London. Bolton C. Flynn R. Harvey E. Morris J. (2004) Assessment of pad clogging.

Journal of Community Nursing. 18(6), 18-20.

Cole L. Nesbitt C. (2004) A three-year multi-phase pressure ulcer prevalence / incidence study in a regional referral hospital. Ostomy and Wound Management. 50(11), 33-40.

Johnson AW. (2004) Overview: Fundamental skin care-protecting the barrier. Dermatologic Therapy. 17, 1-5.
Korting, H.C., Braun-Falco, O. (1996) The Effect of Detergents on Skin pH and Its Consequences

Clinics in Dermatology:; 14: 23-27.

Lyder CH. Shannon R. Empleo-Frazier O. McGeHee D, White C. (2002) A comprehensive program to prevent pressure ulcers in long-term care: exploring costs and outcomes. Ostomy and

Wound Management. 48(4), 52-62.

Millis, A.J., Hoyle, M., McCue, H.M., Martini, H. (1992) Differential expression of metalloproteinase and tissue inhibitor of metalloproteinase genes in aged human fibroblasts. Experimental Cell Research. 201, 73-379.

Timby BK (1996) Hygiene. Fundamental skills and concepts in patient care (6th Ed). Lippincott Raven, London.

Voegeli, D. (2003) Age related changes in the skin. Dermatological Nursing. 2(4), 5-6.

Voegeli D. (2008) The effect of washing and drying practices on skin barrier function. Journal of Wound Ostomy and Continence Nursing. 35(1), 84-90.

Waller, JM., Maibach, HI. (2005) Age and skin structure and function, a quantitative approach (I): blood flow, pH, thickness, and ultrasound echogenicity. Skin Research and Technology.

\section{1, 221-235}

Wound Ostomy and Continence Nurses Society (2003) Guidelines for prevention and management of pressure ulcers 14, WOCN Clinical Practice Guideline Series, WOCN Society, Glenview, Ill.

Figure 1: Diagramatic representation of skin structure. (Taken from: Voegeli D. (2010) Care or Harm: exploring essential components in skin care regimens. British Journal of Nursing. 19(13), 810-819.)

Figure 2: Model of the basic components of skin care (adapted from Johnson, 2004)

Table 1: Some barrier products listed in BNF.

Taken from: Voegeli (2010) British Journal of Nursing, 2010, 19, (13), 818.

\section{KEY POINTS}

Skin care is an important yet largely neglected aspect of care

Good skin care consists of four aspects: Cleansing; hydrating; protecting; replenishing

The range of products available can add to the confusion and often lack good quality evidence of effectiveness

The adoption of clear skin care protocols can have a significant effect on patient outcomes 